CARDIOVASCULAR MEDICINE

\title{
Serial changes in plasma brain natriuretic peptide concentration at the infarct and non-infarct sites in patients with left ventricular remodelling after myocardial infarction
}

\author{
A Hirayama, H Kusuoka, H Yamamoto, Y Sakata, M Asakura, Y Higuchi, H Mizuno, \\ K Kashiwase, Y Ueda, Y Okuyama, M Hori, K Kodama
}

See end of article for authors' affiliations

Correspondence to: Dr Atsushi Hirayama, Cardiovascular Division Osaka Police Hospital, í31 Kitayama-cho, Tennojiku, Osaka, 543-0035 Japan; ahirayama@ oph.gr.jp

Accepted 4 March 2005 Published Online First 17 March 2005

\begin{abstract}
Objectives: To clarify the role of infarct and non-infarct sites on left ventricular (LV) remodelling after myocardial infarction by measuring brain natriuretic peptide (BNP) from each site.

Methods and results: BNP from the aorta and the anterior interventricular vein (AIV) was measured in 45 patients with first anterior myocardial infarction at one, six, and 18 months. The LV was significantly dilated (>10 ml/ $/ \mathrm{m}^{2}$ of end diastolic volume from one to 18 months) in 20 patients (remodelling (R) group) but not in 25 others (non-remodelling (NR) group). Patient characteristics and LV functions did not differ significantly at one month but plasma BNP concentration was higher in group $R$ than in group NR (336 (288) v $116(106) \mathrm{pg} / \mathrm{ml}, \mathrm{p}<0.01$ ), predicting the degree of LV dilatation. The difference in BNP concentration between the aortic root and AIV $(\triangle B N P)$, reflecting $B N P$ secreted from the infarct site, did not differ at one month. In both groups BNP and $\triangle B N P$ significantly decreased from one to six months $(p<0.05)$ and decreased from six months to 18 months, but the change was not significant. BNP and $\triangle B N P$ were significantly higher in group $R$ than in group NR after six months, when LV dilatation was not evident in both groups.

Conclusion: Enhanced BNP secretion at one month in the non-infarct and infarct ventricular sites predicts subsequent LV dilatation (that is, remodelling). The slower process of LV remodelling decreased BNP secretion at both sites. Thus, BNP concentration should be useful for monitoring ventricular remodelling after infarction.
\end{abstract}

W $\mathrm{e}^{1}$ and others ${ }^{2}$ previously reported that synthesis and secretion of brain natriuretic peptide (BNP) was augmented before progressive ventricular dilatation after myocardial infarction and that plasma BNP concentration predicted magnitude of subsequent left ventricular (LV) dilatation. ${ }^{3}{ }^{4}$ Plasma BNP concentration thus reflects LV wall stress leading to dilatation. The difference in plasma BNP concentration between the aortic root and the anterior interventricular vein (AIV) also increases, indicating increased wall stretch at the infarct site. ${ }^{5}$

LV dilatation after myocardial infarction results from both regional wall expansions at the infarct site and volume overload-type hypertrophy in non-infarct myocardium in response to increased wall stress. ${ }^{6}$ Thus, simultaneous measurement of BNP secretion from the infarct site and from the entire ventricle would help to determine mechanisms of LV dilatation. Regional wall stress along with LV dilatation would decline after healing of the infarct. Sequential changes in plasma BNP concentration measured at infarct and non-infarct sites would clarify the role of each site in long term LV remodelling.

Over 18 months, we studied relations between concentrations of BNP, especially BNP at the infarct site, and LV dilatation in patients with their first anterior myocardial infarction.

\section{PATIENTS AND METHODS Patients}

Of 102 patients admitted to our coronary care unit with their first anterior myocardial infarction who received successful reperfusion by primary coronary angioplasty with or without stenting, 45 fulfilled the study inclusion criteria: age below 80 years; absence of cardiomyopathy, severe valve disease, ventricular septal defect, atrial fibrillation, or cardiogenic shock (systolic pressure $<80 \mathrm{~mm} \mathrm{Hg}$ ); follow up cardiac catheterisation and measurement of plasma BNP at one, six, and 18 months after the onset; and no further cardiac events such as unstable angina, myocardial infarction, or silent occlusion of an infarct related artery during the study period. Anterior myocardial infarction was defined by ECGs showing ST segment elevation in at least two contiguous precordial leads and was confirmed by coronary angiograms depicting a total or subtotal proximal occlusion of the left anterior descending coronary artery and subsequent increase of creatine kinase activities more than twice normal range. Of these, we reported on 39 patients previously. ${ }^{1}$ The protocol was approved by the ethics committee of Osaka Police Hospital.

\section{Cardiac catheterisation and analysis of left ventriculography}

Cardiac catheterisation was performed at one month (25-36 days), six months (5-9 months), and 18 months (16-23 months) after onset, with written informed consent at the time of each procedure. Details of cardiac catheterisation were described previously. ${ }^{1}$ Briefly, haemodynamic parameters

Abbreviations: $\mathrm{ACE}$, angiotensin converting enzyme; AIV, anterior interventricular vein; BNP, brain natriuretic peptide; EDVI, end diastolic volume index; LV, left ventricular 
including mean right atrial pressure, LV end diastolic pressure, and systemic blood pressure were measured. Blood was collected simultaneously from the aortic root and AIV for measurement of plasma BNP. To collect blood from the AIV, a 5 French NIH catheter with end hole was placed in the coronary sinus through the brachial vein and then advanced to the AIV with a guidewire. Blood from the AIV and from the aortic root was sampled simultaneously. After completion of haemodynamic measurements and blood sampling, left ventriculography was performed. Lastly, coronary angiography was performed after intracoronary administration of glyceryl trinitrate $(0.1-0.3 \mathrm{mg})$. Left ventriculography was performed in the $30^{\circ}$ right anterior oblique position with a power injection and analysed as described previously. ${ }^{7}$ Briefly, endocardial contours of the LV at end diastole and end systole were traced. The LV volume at these times was measured by the area-length method $^{8}$ and the ejection fraction was calculated. ${ }^{9}$ Regional LV function was assessed by centre line methods as described previously..$^{10}$ Briefly, the centre line was defined as the point midway between the end diastolic and end systolic endocardial contours. Motion was measured along 100 chords within the LV shape constructed perpendicularly to the centre line and plotted in units of SD of normal data based on ventriculograms obtained in 100 normal subjects. Regional wall motion abnormality in the centre of the infarct region was calculated by averaging the motion of chords within the most hypokinetic half of the infarct related territory (chords 10 to 66) and expressed as SD per chord. A hypokinetic area was calculated where motion was depressed by more than 2 SD below the normal mean, expressed as the percentage of endocardial contour length.

\section{Thallium-201 single photon emission computed tomography}

Myocardial single photon emission computed tomography was performed at about one month (19-38 days) after onset of myocardial infarction with a large field of view rotating double crystal gamma camera (OPTIMA, GE Medical, Minneapolis, Minnesota, USA) equipped with a low energy general purpose collimator and a computer interface (Starcam 4000; GE Medical). Image acquisition commenced in the $30^{\circ}$ right anterior oblique projection about three hours after the injection of $114 \mathrm{MBq}{ }^{201} \mathrm{Tl}$. Thirty two sequential images separated by $5.6^{\circ}$ intervals were acquired over $180^{\circ}$. Data were stored in a $64 \times 64$ matrix for subsequent analysis.

Infarct size was assessed according to methods previously described. ${ }^{7}$ Briefly, mean defect severity was determined from the area under the circumferential curve more than 2 SD below normal at each slice. Curves were obtained by dividing the image of the LV into six layers at equidistant intervals perpendicular to its long axis. Defect volume was defined by summation of mean defect severity in each slice and was expressed in arbitrary units.

\section{Measurement of BNP}

All blood samples were collected in a chilled plastic syringe and transferred to chilled siliconised disposable tubes containing aprotinin and EDTA. The plasma fraction was immediately separated at $4^{\circ} \mathrm{C}$ and stored at $-80^{\circ} \mathrm{C}$ until analysis. Plasma natriuretic peptide concentrations were measured by a radioimmunoassay kit specific for BNP (Shionoria, Osaka, Japan). ${ }^{11}$ The lower limit of sensitivity for BNP in this assay was $4.0 \mathrm{pg} / \mathrm{ml}$. Intra-assay and interassay variances were $4.4 \%$ and $2.0 \%$, respectively.

\section{Statistical analysis}

All data were analysed by SPSS version 11.0 (SPSS Inc, Chicago, Illinois, USA). Data are expressed as mean (SD). For continuous variables, differences between groups were assessed by Student's $t$ test; for categorical variables, Fisher's exact test was used. One way analysis of variance was used to assess differences in variables between one, six, and 18 months and two way analysis of variance was used to compare the groups during the study period. For the value of BNP, a non-parametric test was applied. The Kruskal-Wallis test was used to compare more than two groups. Two way analysis was done according to the Friedman's method. Multivariate linear regression analysis was used to identify the independent factors determining the degree of LV increment during the study period. Results were considered significant when the $\mathrm{p}<0.05$.

\section{RESULTS}

\section{Patient characteristics}

Among 45 patients enrolled in this study, 20 patients (remodelling ( $\mathrm{R})$ group) had significant LV dilatation,

\begin{tabular}{|c|c|c|c|}
\hline Variable & NR group $(n=25$ ) & $R$ group $(n=20)$ & $p$ Value \\
\hline Age (years) & $62(9)$ & $63(9)$ & NS \\
\hline Men & $23(92 \%)$ & $15(75 \%)$ & NS \\
\hline Time to reperfusion after onset of infarction (hours) & $12.6(16.3)$ & $18.3(23.3)$ & NS \\
\hline Systemic hypertension & $5(20 \%)$ & $5(25 \%)$ & NS \\
\hline Diabetes mellitus & $7(28 \%)$ & $1(5 \%)$ & NS \\
\hline Hyperlipidaemia* & $14(56 \%)$ & $14(70 \%)$ & NS \\
\hline \multicolumn{4}{|l|}{ Medications } \\
\hline ACE inhibitors & $12(48 \%)$ & $17(85 \%)$ & $<0.05$ \\
\hline Nitrates & $20(80 \%)$ & $12(60 \%)$ & NS \\
\hline Calcium channel antagonists & $6(24 \%)$ & $6(30 \%)$ & NS \\
\hline Diuretics & $2(8 \%)$ & $9(45 \%)$ & $<0.05$ \\
\hline$\beta$ Blockers & $3(12 \%)$ & $0(0 \%)$ & NS \\
\hline Antiplatelet agents & $25(100 \%)$ & $20(100 \%)$ & NS \\
\hline \multicolumn{4}{|l|}{ Angiographic characteristics } \\
\hline Coronary arteries with $>75 \%$ narrowing & & & NS \\
\hline 0 & $7(28 \%)$ & $6(30 \%)$ & \\
\hline 1 & $13(52 \%)$ & $8(40 \%)$ & \\
\hline 2 & $4(16 \%)$ & $5(25 \%)$ & \\
\hline 3 & $1(4 \%)$ & $1(5 \%)$ & \\
\hline Defect volume (arbitrary units) & 938 (708) & $1385(832)$ & NS \\
\hline
\end{tabular}

Data are mean (SD) or number (\%).

*Defined as serum total cholesterol concentration $>5.69 \mathrm{mmol} / \mathrm{l}$ or triglycerides $>1.95 \mathrm{mmol} / \mathrm{l}$.

$\mathrm{ACE}$, angiotensin converting enzyme; NS, not significant. 
Table 2 Left ventricular function and haemodynamic variables in NR and R groups during the study period

\begin{tabular}{|c|c|c|c|c|c|c|}
\hline & \multicolumn{3}{|l|}{ NR group } & \multicolumn{3}{|l|}{$R$ group } \\
\hline & 1 month & 6 months & 18 months & 1 month & 6 months & 18 months \\
\hline Days after index event & $30(7)$ & 201 (49) & $620(115)$ & $32(5)$ & $215(35)$ & 550 (119) \\
\hline Ejection fraction $(\%)^{*}$ & $53(15)$ & $52(14)$ & $50(14)$ & $44(16)$ & $42(14)$ & $44(13)$ \\
\hline EDVI $\left(\mathrm{ml} / \mathrm{m}^{2}\right)^{*}$ & $63(17)$ & $62(21)$ & $58(18)$ & $61(16)$ & $77(22) \dagger$ & $84(22) \dagger$ \\
\hline ESVI $\left(\mathrm{ml} / \mathrm{m}^{2}\right)^{*}$ & 31 (15) & $31(17)$ & 31 (15) & $35(17)$ & $47(22)$ & $49(21) \dagger$ \\
\hline RWM abnormality (SD/chords)* & $-2.98(1.23)$ & $-2.84(1.28)$ & $-2.93(1.26)$ & $-3.34(1.09)$ & $-3.40(0.97)$ & $-3.39(0.79)$ \\
\hline Hypokinetic area $(\%)^{*}$ & $37(19)$ & $34(20)$ & 30 (19) & $48(22)$ & $52(19)$ & $49(16)$ \\
\hline Heart rate (beats $/ \mathrm{min}$ ) & $74(14)$ & $72(17)$ & $72(15)$ & $82(17)$ & $75(14)$ & 70 (11)† \\
\hline Systolic aortic pressure $(\mathrm{mm} \mathrm{Hg})$ & $137(26)$ & $135(29)$ & $144(20)$ & $131(35)$ & $134(37)$ & $143(35)$ \\
\hline LVEDD $(\mathrm{mm} \mathrm{Hg})$ & $10(4)$ & $11(5)$ & $11(5)$ & $14(6)$ & $15(8)$ & $12(5)$ \\
\hline $\operatorname{MAP}(\mathrm{mm} \mathrm{Hg})$ & $4(2)$ & $4(3)$ & $4(3)$ & $4(1)$ & $4(5)$ & $4(6)$ \\
\hline
\end{tabular}

defined as an increase in end diastolic volume index (EDVI) of at least $10 \mathrm{ml} / \mathrm{m}^{2}$ between $1-18$ months after the onset, and 25 patients did not (non-remodelling (NR) group). No significant differences were evident between these two groups in terms of age, sex distribution, presence of established risk factors (diabetes mellitus, hyperlipidaemia, and hypertension), time from onset to reperfusion, angiographic characteristics, or defect volume. Medications given from immediately after admission through to 18 months did not differ between the groups, except for angiotensin converting enzyme (ACE) inhibitor and diuretics, which were taken by significantly more patients in group $\mathrm{R}$ than in group NR (table 1). No patients had coronary intervention besides the acute phase during the study period.

\section{Changes in LV function over time}

Table 2 presents data concerning LV function. No significant differences in LV end diastolic volume, end systolic volume, ejection fraction, regional wall motion abnormality, percentage hypokinetic area, or haemodynamic variables were evident between the two groups at one month. LV end diastolic and end systolic volumes increased significantly during the study period in group $\mathrm{R}$. In group NR, no significant increment in end diastolic or end systolic volume was observed. Reflecting this pattern, end systolic and end diastolic LV volumes and ejection fraction differed significantly between the two groups during the study period. Regional wall motion abnormalities during the study period were worse in group $\mathrm{R}$ than in group NR but the differences were not significant. The percentage hypokinetic area in group NR at one month was somewhat smaller than that in group $\mathrm{R}$ and decreased after one month. The percentage

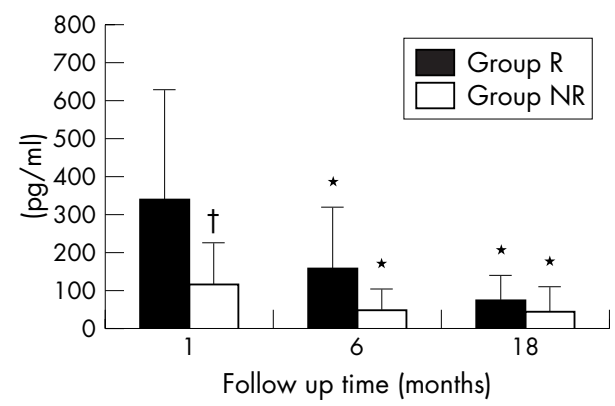

Figure 1 Plasma brain natriuretic peptide (BNP) concentration at the aortic root shown at 1, 6, and 18 months after the onset of myocardial infarction. ${ }^{*} \mathrm{p}<0.05$ within group differences between 1 month and 6 or 18 months; $\uparrow p<0.05$ remodelling $(R)$ group versus non-remodelling (NR) group at 1 month (two way analysis of variance). hypokinetic area in group R, however, did not change; as a result, percentage hypokinetic area during the study period was significantly smaller than in group R. Haemodynamic variables including heart rate, systolic aortic pressure, LV end diastolic pressure, and mean atrial pressure were not significantly different between the two groups or at the follow up times.

\section{Plasma BNP concentration}

Plasma BNP concentration was significantly higher in group $\mathrm{R}(336(288) \mathrm{pg} / \mathrm{ml})$ than in group NR (116 (106) pg/ml) at one month (fig 1). Plasma BNP concentration in group $\mathrm{R}$ decreased significantly from one to six months (152 (162) pg/ $\mathrm{ml})$, then further decreased from six months to 18 months (71 (65) $\mathrm{pg} / \mathrm{ml}$ ), but the difference between six and 18 months did not reach significance. A similar change was observed in group R: plasma BNP concentration in group NR significantly decreased from one to six months (44 (58) pg/ $\mathrm{ml}$ ) and did not change from six months to 18 months (42 (66) $\mathrm{pg} / \mathrm{ml}$ ). Plasma BNP concentrations was significantly higher in group $\mathrm{R}$ than in group NR during the study period.

The difference in plasma BNP concentration between the aortic root and AIV $(\triangle \mathrm{BNP})$, representing the amount of BNP released from the infarct site, was slightly higher in group $\mathrm{R}$ $(665(583) \mathrm{pg} / \mathrm{ml})$ than in group NR (483 (365) pg/ml) at one month, but this was not significant (fig 2). $\triangle \mathrm{BNP}$ in group $\mathrm{R}$ and group NR decreased significantly from one to six months (297 (256) pg/ml for group R; 145 (189) pg/ml for group NR) and decreased from six months to 18 months (190 (207) pg/ $\mathrm{ml}$ for group R, 110 (166) pg/ml for group NR) but the difference was not evident between six months and 18 months, similar to the changes in BNP concentration. $\triangle \mathrm{BNP}$

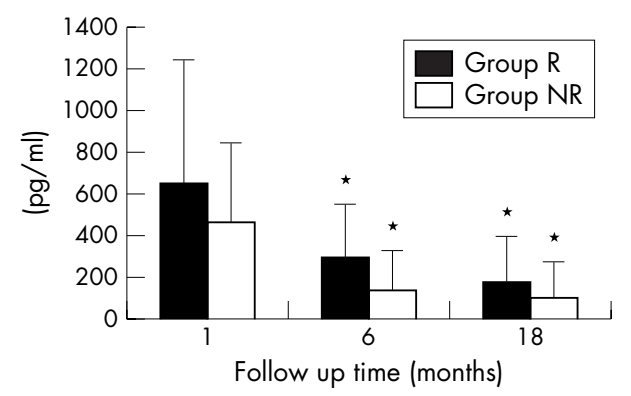

Figure 2 Difference in plasma BNP concentration between the aortic root and the anterior intraventricular vein $(\triangle B N P)$ shown at 1, 6, and 18 months after the onset of myocardial infarction. ${ }^{*} \mathrm{p}<0.05$ within group differences between 1 month and 6 or 18 months (two way analysis of variance). 


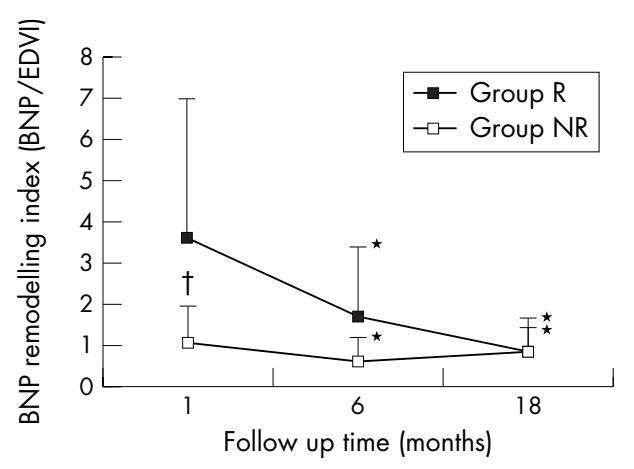

Figure 3 Changes in BNP remodelling index, expressed as BNP/end diastolic volume index (EDVI). ${ }^{*} \mathrm{p}<0.05$ within group differences between 1 month and 6 or 18 months; $\uparrow p<0.05 R$ group versus NR group at 1 month (two way analysis of variance).

was significantly higher in group $\mathrm{R}$ than in group NR at both six months and 18 months.

\section{Relation between plasma BNP concentration and LV dilatation}

To summarise the changes in plasma BNP concentration and in end diastolic volume in both groups, fig 3 shows the BNP remodelling index expressed as BNP/EDVI. In both groups $\mathrm{R}$ and NR, the BNP remodelling index decreased significantly from one month to six months and decreased from six months to 18 months without significant difference. The BNP remodelling index was significantly higher in group $\mathrm{R}$ than in group NR during the entire study period.

\section{Factors predictive of degree of LV dilatation}

An increase in end diastolic volume ( $\triangle E D V I)$ from one to six months was significantly correlated with LV end diastolic pressure $\quad(r=0.422, \quad \mathrm{p}<0.01)$, ejection fraction $(r=-0.322, \quad \mathrm{p}<0.05), \quad$ defect volume $(r=0.391$, $\mathrm{p}<0.01)$, and plasma BNP concentration at one month $(r=0.532, \mathrm{p}<0.001) . \Delta$ EDVI from one to 18 months was significantly correlated with ejection fraction $(r=-0.399$, $\mathrm{p}<0.01)$, regional wall motion abnormality $(r=-0.313$, $\mathrm{p}<0.05)$, percentage hypokinetic area $(r=0.378$, $\mathrm{p}<0.05)$, LV end diastolic pressure $(r=0.369, \mathrm{p}<0.05)$, defect volume $(r=0.460, \mathrm{p}<0.01)$, and plasma BNP concentration at one month $(r=0.436, \mathrm{p}<0.01)$. No significant correlation was noted between $\triangle \mathrm{BNP}$ and $\triangle$ EDVI either from one to six months or from one to 18 months. To elucidate the factors that predict the degree of LV dilatation, multivariate linear regression analysis with incremental method was adopted. In this method, the number of variables in the regression model increases step by step until residual variables are all insignificant. As a result, plasma BNP at one month and diuretics were the variables that correlated significantly with $\triangle$ EDVI from one to six months or from one to 18 months among the factors LV function, haemodynamic variables, age, sex, and medications (ACE inhibitor and diuretics).

\section{DISCUSSION}

Our study shows that secretion of BNP at the infarct segment was augmented after myocardial infarction and gradually decreased independently of subsequent LV dilatation one month after the index event. We also showed that LV dilatation enhanced BNP synthesis at the infarct and noninfarct segments, leading to a significantly high plasma BNP concentration in patients with LV dilatation up to six months; after that, plasma BNP concentration reached a steady state reflecting the decreased rate in the progression of
LV dilatation at 18 months. Furthermore, we confirmed that plasma BNP concentration is a powerful short term predictor of LV dilatation as shown in previous reports, ${ }^{12}$ in addition to long term prediction.

BNP concentration at one month and LV remodelling Increased wall stress after myocardial infarction mostly derives from stretch in the border zone between infarct and non-infarct sites. Here BNP synthesis is presumably enhanced, as shown in experimental animals ${ }^{12}$ and in humans. ${ }^{13}$ BNP synthesis in the infarct area including the border zone, defined as $\triangle \mathrm{BNP}$, should be augmented in response to increased regional wall stress. ${ }^{5} \Delta \mathrm{BNP}$ was slightly higher in patients with than in patients without LV dilatation, but the difference did not attain significance at one month. This may be explained as the balance of the amount of residual viable myocardium synthesising BNP and the wall stress regulating BNP synthesis at the infarct site. The increased wall stress in group R compared with group NR augments BNP synthesis of a residual viable myocytes. On the other hand, residual myocytes at the infarct site, producing a small amount of BNP reflecting less wall stress, may be slightly greater than in group $\mathrm{R}$, although in this study the difference in infarct size was not significant. As a result, the amount of BNP released from the infarct site was essentially similar in the two groups. As over 99\% of circulating BNP was synthesised in the LV after myocardial infarction, ${ }^{5}$ plasma BNP concentration reflects the BNP synthesised at both infarct and non-infarct sites. The significant difference in plasma BNP concentration was evident at that point without a clear cut difference in $\triangle \mathrm{BNP}$, suggesting that higher regional wall stress in the noninfarct sites in patients with LV dilatation enhances BNP synthesis at non-infarct sites.

\section{Change in plasma BNP concentration after one month and $L V$ remodelling}

From one to six months, the concentration of plasma BNP and of BNP released from the infarct site $(\triangle \mathrm{BNP})$ decreased significantly in both groups; however, the concentration of plasma BNP and $\triangle \mathrm{BNP}$ were significantly higher in patients with LV dilatation than in patients without LV dilatation at six months, suggesting that the ventricular wall stress after myocardial infarction is still evident at six months after the onset during LV dilatation. In patients without LV dilatation, functional recovery after fibrotic scar formation may decrease LV wall stress during the first six months resulting in the termination of LV dilatation. On the other hand, in patients with LV dilatation, poor recovery of regional wall motion at the infarct site results in a sustained excess of ventricular wall stress leading to LV dilatation. ${ }^{14}$ 15 Plasma BNP concentration and $\triangle \mathrm{BNP}$ in patients with LV dilatation decreased from six to 18 months but was significantly higher than in patients without LV dilatation. LV dilatation was not evident from six months to 18 months even in patients with LV dilatation. These findings suggest that the slower process of LV remodelling decreased BNP secretion and resulted in insignificant changes in BNP concentration, although further studies with more patients will be necessary.

\section{Prediction of long term LV dilatation}

As previously reported, plasma BNP concentration at one week or one month after the onset of myocardial infarction correlated significantly with the degree of LV dilatation at six months. ${ }^{12}$ In the present study, plasma BNP at one month was significantly correlated with severity of LV dilatation both from one to six months and from one to 18 months. Multiple regression analysis identified plasma BNP concentration as a determinant of $\mathrm{LV}$ dilatation. Besides BNP 
concentration, the use of diuretics was identified as a factor determining LV dilatation. Although all medications were started immediately after admission, diuretics were mainly given to patients with clinical heart failure. The selection bias may be closely related to the identification of factors by multivariate linear regression analysis. These findings indicate that increased wall stress reflected by plasma BNP concentration is the key factor determining future LV function.

\section{Study limitations}

Although this study was limited to patients who were successfully reperfused during the acute phase without new cardiac events including silent restenosis, ischaemia, or reocclusion of the infarct related artery, over an 18 month period from the index event ${ }^{16}{ }^{17}$ not all LV remodelling could be predicted.

We assumed that the difference in BNP concentration between the aorta and the AIV reflects infarct site BNP production. However, some blood may be drained from the infarct site independently from the AIV through minimal veins draining directly into the cardiac cavities, as well as into the rest of the coronary sinus venous system. When this drainage occurs, it may be underestimated by our calculation of BNP production in the infarct site. Nevertheless, the contribution of this error would not be large, and the conclusion is still applicable. Circulating BNP concentration was not affected by the drainage of blood from the infarct site through the AIV because circulating BNP was assumed to be produced in the infarct and the non-infarct LV. BNP can be produced in various parts of the circulation system including the right ventricle, atria, and pulmonary vein in addition to the LV. The amount of BNP produced in these other areas is very low as previously reported ${ }^{51819}$ and did not affect our conclusion.

Our patients were not given medications that are usually administered in western countries; despite the high use of antiplatelets, in this study $\beta$ blocking agents were rarely used. The high incidence of vasospasm after myocardial infarction ${ }^{20}$ or of side effects (for example, bradycardia and hypotension) in Japanese compared with white patients may be why $\beta$ blockers are rarely given. The significantly higher use of ACE inhibitor might have affected the production of BNP in group $\mathrm{R}$, but multivariate linear regression analysis showed that ACE inhibitor was not an independent predictor of LV remodelling. Furthermore, ACE inhibitor has been shown to have little preventive effect on LV dilatation in patients without poor LV function such as patients in group R. ${ }^{21}$ Even if medications used in this study did not relate to the major conclusion, the effect of medication must be considered. However, the number of patients is too small to assess the effects of pharmacological interventions. ${ }^{22}$ Further, a large scale study will be necessary to assess relations between plasma BNP concentration and LV remodelling under a variety of conditions likely to affect the process.

In conclusion, the present study of plasma BNP and $\triangle \mathrm{BNP}$ clarifies the important role of the non-infarct segment in LV remodelling. Furthermore, BNP may prove to be a useful clinical tool to predict remodelling and assess the timing of the process, but this study did not provide this proof.

\section{Authors' affiliations}

A Hirayama, Y Ueda, Y Okuyama, K Kodama, Cardiovascular

Division, Osaka Police Hospital, Osaka, Japan

H Kusuoka, H Yamamoto, Department of Cardiology, Osaka National Hospital, Osaka, Japan

Y Sakata, M Asakura, Y Higuchi, H Mizuno, K Kashiwase, M Hori,

Division of Cardiology, Department of Internal Medicine and

Therapeutics, Osaka University Graduate School of Medicine, Suita,

Japan

\section{REFERENCES}

1 Hirayama A, Yamamoto H, Sakata Y, et al. Usefulness of plasma brain natriuretic peptide after acute myocardial infarction in predicting left ventricular dilatation six months later. Am J Cardiol 2001;88:890-3.

2 Nagaya N, Nishikimi T, Goto Y, et al. Plasma brain natriuretic peptide is a biochemical marker for the prediction of progressive ventricular remodeling after acute myocardial infarction. Am Heart J 1998;135:21-8.

3 Pouleur H, Rousseau MF, van Eyll C, et al. Assessment of regional left ventricular relaxation in patients with coronary artery disease: importance of geometric factors and changes in wall thickness. Circulation 1984;69:696-702.

4 Pfeffer MA, Pfeffer JM. Ventricular enlargement and reduced survival after myocardial infarction. Circulation 1987;75:IV93-7.

5 Sumida $H$, Yasue $H$, Yoshimura $M$, et al. Comparison of secretion pattern between A-type and B-type natriuretic peptides in patients with old myocardial infarction. J Am Coll Cardiol 1995;25:1105-10.

6 McKey R, Pfeffer M, Pasternark R, et al. Left ventricular remodeling after myocardial infarction: a corollary to infarct expansion. Circulation 1986;74:693-702.

7 Hirayama A, Adachi T, Asada S, et al. Late reperfusion for acute myocardial infarction limits the dilatation of left ventricle without the reduction of infarct size. Circulation 1993;88:2565-74.

8 Sandler $\mathrm{H}$, Dodge $\mathrm{H}$. The use of single plane angiography for the measurement of left ventricular volume in man. Am Heart $J$ 1968;75:325-34.

9 Dodge $\mathrm{H}$, Sandler H, Baxley W, et al. Usefulness and limitation of radiographic methods for determining left ventricular volume. Am J Cardiol 1966;18:10-24.

10 Sheehan F, Bolson E, Dodge $H$, et al. Advantages and applications of the centerline method for characterizing regional ventricular function. Circulation 1986;74:293-305.

11 Kono M, Yamauchi M, Tsuji T, et al. An immunoradiometric assay for brain natriuretic peptide in human plasma. Kaku lgaku Gijutsu 1993;13:2-7.

12 Hama N, Itoh H, Shirakami G, et al. Rapid ventricular induction of brain natriuretic peptide gene expression in experimental acute myocardial infarction. Circulation 1995;92:1558-64.

13 Jougasaki M, Yasue H, Mukoyama M, et al. Appearance of atrial natriuretic peptide in the ventricles in patients with myocardial infarction. Am Heart $J$ 1990;119:92-6.

14 Grossman W, Jones D, McLaurin LP. Wall stress and patterns of hypertrophy in the human left ventricle. J Clin Invest 1975;56:56-64.

15 Grossman W. Cardiac hypertrophy: useful adaptation or pathologic process? Am J Med 1980;69:576-84.

16 White $\mathrm{H}$, Cross $\mathrm{D}$, Elliot J, et al. Long-term prognostic importance of patency of the infarct-related coronary artery after thrombolytic therapy for acute myocardial infarction. Circulation 1994;89:61-7.

17 Brodie B, Stuckey T, Kissling G, et al. Importance of infarct-related artery patency for recovery of left ventricular function and late survival after primary angioplasty for acute myocardial infarction. J Am Coll Cardiol angioplasty for ac

18 Murakami Y, Shimada T, Inoue S, et al. New insights into the mechanism of the elevation of plasma brain natriuretic polypeptide levels in patients with left ventricular hypertrophy. Can J Cardiol 2002;18:1294-300.

19 Yasue H, Yoshimura M, Sumida H, et al. Localization and mechanism of secretion of B-type natriuretic peptide in comparison with those of A-type natriuretic peptide in normal subjects and patients with heart failure. Circulation 1994;90:195-203.

20 Pristipino C, Beltrame JF, Finocchiaro ML, et al. Major racial differences in coronary constrictor response between Japanese and Caucasians with recent myocardial infarction. Circulation 2000;101:1102-8.

21 Latini R, Maggioni AP, Flather M, et al. ACE inhibitor use in patients with myocardial infarction: summary of evidence from clinical trials. Circulation 1995:92:3132-7.

22 Pfeffer MA, Braunwald E, Moye LA, et al. Effect of captopril on mortality and morbidity in patients with left ventricular dysfunction after myocardial infarction. Results of the survival and ventricular enlargement trial. NEngl J Med 1992;327:669-77. 